\title{
Philosophiques
}

\section{Le postulat d'objectivité dans la science et la philosophie du sujet}

\author{
G. B. Madison
}

Volume 1, numéro 1, avril 1974

URI : https://id.erudit.org/iderudit/203005ar

DOI : https://doi.org/10.7202/203005ar

Aller au sommaire du numéro

\section{Éditeur(s)}

Société de philosophie du Québec

\section{ISSN}

0316-2923 (imprimé)

1492-1391 (numérique)

Découvrir la revue

\section{Citer cet article}

Madison, G. B. (1974). Le postulat d'objectivité dans la science et la philosophie du sujet. Philosophiques, 1(1), 107-139. https://doi.org/10.7202/203005ar

\section{Résumé de l'article}

La question de fond traitée dans cet article est celle des rapports entre la science et la philosophie. Cette question est abordée par le biais d'une considération de la critique de la science élaborée par la phénoménologie. La philosophie de Merleau-Ponty sert d'exemple de la position phénoménologique. Le but de l'article est de démontrer l'insuffisance de la position de Merleau-Ponty - et, par extension, de la phénoménologie en général - en ce qui concerne son appréciation de la nature véritable du projet scientifique. A cette fin, on essaie de saisir le sens véritable des développements récents dans la science en examinant quatre cas particuliers : la physique nucléaire, la psychologie, la biologie moléculaire, la cybernétique. Le résultat de cette analyse est de montrer que la lecture que faisait Merleau-Ponty des visées et des tendances de la science était erronée et que la position classique de la phénoménologie est indéfendable. Comme, cependant, l'auteur se trouve en accord avec la motivation profonde de Merleau-Ponty et de la phénoménologie en ce qui concerne l'irréductibilité du sujet ou de la conscience à l'objectivisme scientifique, il est alors amené à se demander ce que pourrait être une position phénoménologique satisfaisante vis-à-vis de la science. C'est ainsi que l'article se termine par une critique de toute position positiviste (et structuraliste) en philosophie et par une tentative d'indiquer ce que devrait être la véritable nature du discours philosophique, une fois que celui-ci est purifié de tout empirisme, c'est-à-dire de toute prétention à des connaissances positives.
Ce document est protégé par la loi sur le droit d'auteur. L’utilisation des services d'Érudit (y compris la reproduction) est assujettie à sa politique d'utilisation que vous pouvez consulter en ligne.

https://apropos.erudit.org/fr/usagers/politique-dutilisation/ 


\title{
LE POSTULAT D'OBJECTIVITÉ DANS LA SCIENCE ET LA PHILOSOPHIE DU SUJET
}

\author{
par G.B. Madison
}

IL EXISTE UNE CRITIQUE PHÉNOMÉNOLOGIQUE de la science. Ce qui est critiqué dans la science, c'est son naturalisme, son objectivisme, son déterminisme, ses analyses en termes de causalité, etc. Ce que la phénoménologie voudrait défendre contre ces tendances scientifiques, c'est la primauté du sujet, de la conscience, de l'expérience vécue, l'irréductibilité de la subjectivité à l'objectivité naturaliste. A l'encontre de l'objectivisme scientifique, la phénoménologie est ainsi une philosophie du sujet, et elle veut défendre la subjectivité ou la conscience contre la tendance de la science à l'objectifier, à ne considérer le sujet que comme un objet dans le monde. C'est ainsi que la science est critiquée pour avoir oublié le sujet; elle est critiquée parce qu'elle ignore le point de vue de la subjectivité.

Mais on peut se demander si tout cela constitue vraiment une faute de la part de la science. Même si la science est objectiviste et ignore le sujet, est-ce là vraiment une faute? Et, d'autre part, la phénoménologie a-t-elle vraiment quelque chose à apporter à la science ou, autrement dit, la science a-t-elle quelque chose à apprendre de la phénoménologie? Beaucoup de phénoménologues l'ont cru; ils ont dit que la phénoménologie peut aider la science à se purifier de ses «erreurs » naturalistes ou objectivistes et peut lui enseigner le respect du sujet. Tel est le cas notamment de Husserl et de Merleau-Ponty. La phénoménologie, et elle seule, peut fournir à la science les «fondements » qui lui manquent. L'objectivisme est une erreur, et la science doit s'en rendre compte. La phénoménologie a donc un travail positif à faire du côté de la science, et la science, quand elle se remettra de ses erreurs objectivistes et naturalistes, deviendra «phénoménologique ». 
Que faut-il penser de ce point de vue? Serait-il réellement possible de réconcilier la philosophie du sujet et l'objectivisme scientifique, l'analyse réflexive et l'analyse objective, et de «phénoménologiser » la science? Ou ne faudrait-il pas plutôt soumettre la critique phénoménologique de la science elle-même à une critique? Le temps n'est-il pas venu où, au lieu de réitérer les thèses phénoménologiques classiques, on devrait les soumettre à un regard critique? Les thèses que je propose de développer ici sont les suivantes: $1^{\circ}$ l'objectivisme dans la science, lcin de s'atténuer, s'est plutôt renforcé, $2^{\circ}$ l'objectivisme est la méthode même de la science, la méthode qui lui convient le mieux; la science positive ignore le sujet phénoménologique, et ce n'est pas une erreur ou une omission regrettable de sa part, $3^{\circ}$ le rapport entre phénoménologie ou philosophie du sujet et science ou savoir objectif est donc à revoir et à redéfinir.

Je propose de procéder de la façon suivante. D'abord (section I), je présenterai la position phénoménologique à l'égard de la science telle qu'on la trouve chez Merleau-Ponty, position, pourrait-on dire, classique. Ensuite (section II), je jetterai un rapide coup d'oeil sur l'évolution de la science elle-même avec l'intention de montrer que le développement effectif de la science semble être en fait contraire à ce qu'aurait exigé et espéré la phénoménologie. Je me bornerai ici à de brèves remarques sur quatre cas particuliers: (1) la physique nucléaire, (2) la Gestaltpsychologie, (3) la biologie, (4) la cybernétique. Ensuite (section III), je tâcherai, à la lumière de cette analyse, d'éliminer certaines équivoques encore présentes aujourd'hui dans les thèses phénoménologiques à l'égard de la science. Enfin (section IV), je suggérerai en guise de conclusion que la science peut fort bien être objectiviste et positiviste, mais que cet état de choses ne justifie tout de même pas une philosopbie objectiviste et positiviste. La philosophie du sujet aurait toujours une tâche importante à remplir, mais seulement à condition qu'elle se distingue nettement de l'analyse scientifique et renonce une fois pour toutes à joindre le point de vue objectif et le point de vue réflexif. C'est alors, et alors seulement, qu'elle aurait des chances de retrouver sa propre voix. 
Merleau-Ponty croyait discerner une sorte de convergence entre la science et la phénoménologie, et c'est à la lumière de cette conviction que sa critique de la science doit être comprise, en particulier ce qu'il dit au sujet de la psychologie. Sa position pourrait se résumer ainsi: les recherches en psychologie l'ont amené à abandonner dans la pratique la pensée causale; à l'ancien associationnisme mécaniste la nouvelle psychologie a substitué la notion de forme ou de structure significative. Seulement la Gestaltpsychologie n'a pas été suffisamment radicale car, au lieu de tirer les conséquences théoriques nécessaires de cette notion de forme, à savoir l'abandon complet de la pensée causale et objective, elle persiste à vouloir interpréter la forme ou le gestalt objectivement. C'est-à-dire que les psychologues n'ont pas compris 《la portée philosophique de leur méthode. ${ }^{1} \mathrm{La}$ seule faute de la Gestaltpsychologie est de ne pas avoir rompu avec le naturalisme. De ce fait «elle devient infidèle à ses propres descriptions ${ }^{2}$; «elle oublie ses descriptions les plus valables quand elle cherche à se donner une charpente théorique. ${ }^{3} \mathrm{Ce}$ qui manque donc à la Gestaltpsychologie, c'est

un renouvellement des catégories: elle en a admis le principe, elle l'a appliqué à quelques cas particuliers, elle ne s'aperçoit pas que toute une téforme de l'entendement est nécessaire si l'on veut traduire exactement les phénomènes et qu'il faut pour y parvenir remettre en question la pensée objective de la logique et de la philosophie classiques, mettre en suspens les catégories du monde, mettre en doute, au sens cartésien, les prétendues évidences du réalisme, et procéder à une véritable 《réduction phénoménologique 》. ${ }^{4}$

En un mot, si la Gestaltpsychologie veut accorder à ses découvertes leur pleine valeur, elle doit mettre explicitement en oeuvre la réduction phénoménologique. Pour Merleau-Ponty « La

1. M. MERLEAU-PONTY, Pbénoménologie de la perception, Paris, Gallimard, 1945, p. 58.

2. Ibid.

3. Ibid., p. $62 \mathrm{n}$.

4. Ibid., p. 60 . 
psychologie de la forme a pratiqué un genre de réflexion dont la phénoménologie de Husserl fournit la théorie. ${ }^{5}$ La forme perceptive n'est pas une chose objective, existant dans le monde, c'est une structure qui n'a de sens que pour un sujet qui le vit. ${ }^{6}$ On ne peut donc, même dans la science, ignorct le sujet qui vit ses expériences, et l'important donc, c'est que la science abandonne la pensée causale et objectiviste et adopte le point de vue du sujet, le point de vue phénoménologique. En somme, pour Merleau-Ponty, la description psychologique doit se purifier de tout psychologisme et de tout objectivisme, et c'est alors qu'elle pourrait « devenir une méthode philosophique. ${ }^{7}$

La position de Merleau-Ponty est donc assez nette. D'une part, il critique la science parce qu'elle est objective et parce qu'elle ignore le sujet phénoménologique. C'est à cet objectivisme qu'il s'oppose. Mais, d'autre part, il souhaite une réconciliation entre philosophie et science, une réconciliation qu'il tient pour possible à condition que la science renonce à vouloir tout expliquer du seul point de vue objectif et reconnaisse la primauté de l' «expérience». A cette condition la science «redeviendra philosophie $\gg{ }^{8}$

Or il me semble que la position de Merleau-Ponty, position partagée par beaucoup de phénoménologues, s'appuie sur une fausse conception de la science et que, par conséquent, les relations qu'il voit entre philosophie et science sont tout aussi erronées. A force de vouloir opérer un rapprochement entre philosophie et science, Merleau-Ponty est amené à tout simplement méconnaître la véritable nature du projet scientifique. Quand il dit que la psychologie oublie et «devient infidèle à ses propres descriptions » lorsqu'elle essaie d'en donner une charpente théorique, il semble ignorer qu'il est essentiel à toute science de ne pas se contenter uniquement d'une description des phénomènes, mais qu'il faut essayer de former des modèles qui puissent expliquer objectivement ces phénomènes. Et cette explication sera toujours ob-

5. Ibid., p. 62.

6. Cf. Ibid., p. 139-40.

7. Ibid., p. 77.

8. L'oeil et l'esprit, Paris, Gallimard, 1964, p. 13. 
jective et réductrice en ce sens qu'elle montrera que ce qui est phénoménologiquement apparent n'est que la conséquence, l'effet, de mécanismes plus profonds. A partir des phénomènes constatés, l'explication scientifique et la compréhension phénoménologique empruntent deux chemins totalement divergents.

Il me semble d'ailleurs que les développements récents dans la science montrent assez clairement que la science peut fort bien abandonner son vieil empirisme et mécanisme dogmatiques sans pour autant se rapprocher des notions de sens et de comprébension telles qu'elles sont comprises par la phénoménologie. En d'autres termes, il y a eu dans la science certaines révisions conceptuelles qui ont eu pour effet de la rendre d'autant plus résistante aux critiques de la phénoménologie et qui n'ont fait que la renforcer comme pensée objective. La science, il me semble, ne s'est nullement trouvée obligée d'abandonner sa demande d'objectivité ou son postulat d'objectivité, comme dirait J. Monod, en faveur du point de vue du sujet, de la conscience réflexive, de l'expérience vécue. C'est ce que je vais essayer de suggérer maintenant en passant rapidement en revue quatre cas particuliers, après quoi il faudra reprendre la question des rapports entre philosophie du sujet et science objective.

(1) Quand la phénoménologie critiquait la notion de causalité dans la science, ce qu'elle contestait, c'est l'idée d'un déterminisme objectif et la notion d'une nature parfaitement déterminée en soi. C'est ce que Merleau-Ponty appelle «le préjugé de l'être déterminé ou du monde ${ }^{9}{ }^{9}$ C'est l'idée défendue par le mécanisme déterministe classique que si l'on pouvait déterminer la position de tous les éléments d'un système et la loi de leurs mouvements, on pourrait prédire exactement ce que sera un état futur de ce système. Or, ce qui est intéressant dans le cas de la PHYSIQUE DES QUANTA, c'est que, pour des raisons exclusivement scientifiques (nécessité de rendre compte d'un état de choses cons-

9. Pbénoménologie de la perception, p. 62. 
taté et vérifié expérimentalement), la physique atomique en est venue à rejeter elle-même la notion d'un déterminisme, d'une nature en soi, et ceci, sans aucun abandon de la notion de causalité.

Comme dans le cas de la Théorie de la Relativité, la nouvelle physique nucléaire ne prétend nullement être un rejet de la physique classique. Seulement, elle en limite la portée et surtout elle se débarasse de beaucoup de présupposés de nature proprement métaphysique, c'est-à-dire non-scientifique, que l'ancienne physique comportait malgré tout et, au premier chef parmi ceuxci, son réalisme naif. Comme le dit très bien C.F. von Weizsäcker, "The object of the critique of quantum mechanics is not, therefore, the concept of the causal nexus, but that of the 'thing or event in itself'. Thus we should rather speak of a critique of the category of substance. ${ }^{10}$ Mais comme le remarque aussi von Weizsâcker, cette façon de parler est ambiguë. "We had better say: Quantum mechanics recognizes the relational character of the categories. Substance, causality, etc., designate not realities in themselves, but realities known by men. »

Ceci est très important. La physique nouvelle ne rejette pas les notions de causalité, de substance et d'objectivité, elle ne fait que les relativiser. Weizsâcker parle d'un «collapse» ou d'une «failure » des catégories de substance et d'objectivtié, mais cette façon de parler est effectivement ambiguë, et il ne faut surtout pas se tromper là-dessus. Ce qui s'est passé, c'est que la physique nouvelle a enlevé ces notions de causalité, de substance, etc., de leur contexte métaphysique où elles se définissaient par rapport à la notion d'une nature absolue, en soi, pour les redéfinir scientifiquement, c'est-à-dire exclusivement par rapport au contexte expérimental. ${ }^{11}$ En devenant des notions non plus absolues mais fonctionnelles ou opérationnelles, elles ne cessent pas pour autant d'être objectives. ${ }^{12}$ Weizsäcker dit que la différence entre la physique des quanta et la physique classique ne concerne pas le

10. C.F. VON WEIZSAKER, The World View of Physics, London, Routeledge and Kegan Paul, 1952, p. 88.

11. "The science of today is operational; that is, it consider every statement as e-sentially concerned with possible experiments or observable processes 》. N. WEINER, Cybernetics, Cambridge, Mass., The M.I.T. Press, 1961, p. 125.

12. «In those realms of nature which cannot be observed immediately, the 
principe de causalité en tant que tel, mais seulement son applicabilité qui se trouve maintenant limitée. ${ }^{13}$ Encore une façon de parler. La causalité dans la physique moderne n'est pas moindre, elle est différente. Elle est maintenant comprise dans un sens non absolu et dogmatique, mais opérationnel. C'est le sens du fameux principe d'incertitude ou, comme on le dit si bien en anglais, «the principle of interderminacy».

Ce principe ne signale pas qu'une brèche a été ouverte dans un rigide déterminisme naturel par où le philosophe pourrait faire entrer son libre arbitre. Ce n'est pas une concession faite à la philosophie du tout. Ce principe ne fait que redéfinir la notion même de causalité, qui ne se refère plus à une inconnaissable nature en soi, mais à une situation expérimentale. La notion de causalité est redéfinie en termes fonctionnels, c'est-à-dire en termes de statistique et de probabilité.

Et en fait - et c'est ce qu'il y a d'intéressant - on pourrait même dire que la notion de causalité ne reçoit son plein sens que dans la nouvelle physique, dans un contexte non métaphysiquement déterministe mais probabiliste. Weizsâcker dit : "Even in quantum mechanics a broadened 'causality' is still valid if we admit that mathematically formulated probability relationships fall under this concept. ${ }^{14}$ Mais le célèbre mathématicien $\mathrm{N}$. Wiener, «père» de la cybernétique, est beaucoup plus catégorique :

In a rigid system, without degrees, if we introduce causation it pervades the whole system, and the only conceivable cause of any future status is the entire past. But in a world in which the whole past causes the whole future in an integral inseparable way, the category of cause has no operational significance. In such a system, the introduction of purpose anywhere will again be all-inclusive and the notion will again be operation-

scientific law becomes more and more only an indication in regard to the possibility and the result of experiments; a law concerning our ability to produce phenomena can be produced. But what has been objectively determined here is no longer a being, but a give-and-take between acting and perceiving ». Weizsäcker, op. cit., p. 200.

13. Ibid., p. 99.

14. Ibid., p. 125 . 
nally meaningless. (...) The concept of cause is only significant when there are different degrees of causation that can be measured. To give causation degrees means to correlate changes in initial conditions with the corresponding changes in later conditions. This requires the entire apparatus of statistics and probability. (...) Modern science has indeed introduced degrees, and has thus justified the realization of experiments and has made possible the significant use of the term cause $\ldots{ }^{15}$

Loin donc d'abandonner la causalité comme mode d'explication, la science moderne ne fait que la renforcer: comme le dit Wiener, elle a rendu possible l'usage significatif de ce concept. Ce serait aussi une erreur que de penser que la science moderne, en se libérant de ses vieux préjugés métaphysiques, se rapproche de la phénoménologie et de son analyse acausale. Quand Heisenberg dit que :

Les tenants de l'atomisme ont dû se rendre à cette évidence que leur science n'est qu'un maillon de la chaîne infinie de dialogues entre l'homme et la nature et qu'elle ne peut plus parler simplement d'une nature «en soi». Les sciences de la nature présupposent toujours l'homme et comme l'a dit Bohr, nous devons nous rendre compte que nous ne sommes pas spectateurs mais acteurs dans le thêâtre de la vie. ${ }^{16}$

et quand Weizsãcker, parlant du Je et du monde, dit «both active and suffering at once, become what they are only in the interaction with their opposite pole $»,{ }^{17}$ il est peut-être normal que les phénoménologues se trompent. Le physicien n'est-il pas en train d'abandonner son objectivisme, le point de vue du spectateur étranger, comme le dit Merleau-Ponty, pour adopter le point de vue transcendental, réflexif ? ${ }^{18}$

15. A. ROSENBLUETH AND N. WIENER, «Purposejul and Non-Purposeful Behavior 》, Pbilosopby of Science, t. 17, no. 4, 1950, p. 320.

16. W. HEISENBERG, La nature dans la physique contemporaine, Paris, Gallimard, collection «Idées», 1962, p. 18-19.

17. WEIZSACKER, op. cit., p. 57.

18. Je cite le texte suivant à titre d'exemple de ce point de vue. «La philosophie transcendentale reconnaît que l'être-conscient est le phénomène fondamental, absolument indépassable. (...) Dans la physique moderne on 
Mais en fair si la physique insiste beaucoup sur le rapport entre l'objet et le sujet, ce n'est nullement pour défendre le point de vue de la conscience transcendentale, de la constitution ou de l'analyse intentionnelle. La logique à l'oeuvre ici poursuit un tout autre but, ce but étant l'objectification de ce rapport lui-même. On n'a plus une nature en soi, mais on n'a pas non plus un sujet transcendental pour qui le monde existe. Ce qu'on a, c'est un système objectif qui inclut l'objet aussi bien que le sujet. Autrement dit, si la nouvelle physique a effectivement reconnu le sujet, ce sujet n'est pas l'ego transcendental de Husserl ou le sujet incarné de Merleau-Ponty mais un sujet, pour ainsi dire, objectif, réductible à une formule mathématique. Tout ceci est évidemment aux antipodes de la phénoménologie. Le monde des particules atomiques n'est pas le monde vécu, et toute tentative visant à faire converger la physique et la phénoménologie, comme si elles disaient la même chose en deux langues différentes, ne pourrait se faire que par, soit une ignorance de la nature réelle des développements dans la science, soit par une arrogance philosophique qui, prétendant pouvoir dégager une «philosophie implicite » dans la science, prétend aussi pouvoir interpréter le sens ultime de ses concepts opératoires mieux qu'elle ne saurait le faire elle-même. Je reviendrai d'ailleurs sur cette dernière question dans la section suivante.

(2) L'évidence commence donc à s'imposer. Le processus à l'oeuvre dans la science contemporaine, n'est-il pas une tendance vers un renforcement de l'objectivisme et non vers son atténuation? Au lieu d'avoir abondonné son naturalisme, son objectivisme, son mécanisme, n'est-ce pas le cas que la science n'a fait que les transformer? Il y a eu transmutation d'un objectivisme substantialiste et naivement réaliste en objectivisme fonctionnel et statistique. Ce n'est peut-être pas un hasard qu'on appelle la nouvelle physique mécanique quantique (quantum mechanics).

retrouve donc ce sue la philosophie transcendentale connaissait depuis tout au moins Fichte et Hegel, c'est-à-dire que l'objet existant objectivement pour soi n'est qu'une fiction. (...) Ainsi la caractère transcendant de l'objet est absorbé définitivement dans la structure transcendentale du sujet ». P.K. SCHNEIDER, «Science, Cybernétique et Conscience», Les Archives de pbilosopbie, t. xxx, no. iii, 1967, p. 332-33. 
Car la science serait toujours aussi mécaniste, mais le mécanisme moderne serait plutôt, pour ainsi dire, structuraliste. Si tel est bien le cas, ce développement n'est pas sans ironie. Car ce que la science contemporaine aurait ainsi réalisé pour elle-même et par ses propres moyens, c'est le programme de Merleau-Ponty lui-même, à savoir un dépassement des antimonies traditionnelles entre réalisme et idéalisme, entre empirisme et intellectualisme. Ayant jeté par-dessus bord la notion d'un réel parfaitement déterminé en soi, la science n'a pas pour autant embrassé l'idéalisme traditionnel, elle ne conçoit pas la nature comme simple produit d'une conscience souveraine. Mais «ce nouveau mode d'analyse qui dépasse les alternatives classiques de l'empirisme et de l'intellectualisme » - pour employer une expression qu'utilise Merleau-Ponty pour désigner sa propre position - ce nouveau mode d'analyse de la science ne ressemble nullement à la troisième alternative que propose Merleau-Ponty, à savoir une «analyse existentielle ${ }^{19}$ qui met la priorité sur une expérience et une signification vécues. ${ }^{20} \mathrm{La}$ troisième voie de la science contemporaine va plutôt en un sens exactement inverse, l'analyse structurale ou fonctionnelle de la science n'est pas une redécouverte de l'«expérience » mais bien plutôt sa naturalisation. Voilà donc l'ironie de la chose.

Maintenant, est-ce qu'on ne peut pas dire que c'est justement ce phénomène qui s'est produit dans le cas de la PSYCHOLOGIE? On sait que les premiers psychologues de la Forme avaient été effectivement inspirés par la phénoménologie de Husserl, mais est-ce là un fait vraiment significatif pour comprendre l'épistémologie interne de cette science? Ou n'est-ce pas plutôt le cas que la phénoménologie a été simplement une occasion pour la psychologie de se développer selon sa propre logique? Si les Gestaltistes ont été influencés par Husserl, c'est aussi un fait qu'ils n'en ont pas «compris» ou retenu grand'chose, à part l'idée qu'il existe un rapport étroit entre sujet et objet. Et ce n'est pas un fait sans intérêt non plus que Husserl, dans la Nachwort à ses Ideen, ait sévèrement critiqué et l'ancienne psychologie atomiste et la Gestalt-psychologie pour leur naturalisme.

19. Pbénoménologie de la perception, p. 158.

20. Cf. Ibid., p. 140. 
La notion de Gestalt ou de forme significative ne serait pas ainsi une reconnaissance, même implicite, de la primauté du sujet et de l'expérience vécue, mais représenterait plutôt une réforme à l'intérieur d'une psychologie objective. Comme dans le cas de la physique, la psychologie a transformé - en l'améliorant - le statut d'objectivité. A la place du vieil associationnisme atomiste, elle a substitué une analyse dynamique et structurale. La notion de structure a depuis lors fait son chemin dans la psychologie et on a aujourd'hui, parmi d'autres, une psychologie génétique structurale (Piaget) qui est non seulement non-phénoménologique mais même farouchement anti-phénoménologique.

(3) Il va sans dire que le phénomène de la psychè est étroitement lié au phénomène de la vie. Voilà donc un autre domaine où la philosophie se sent directement concernée. Exactement comme il existe une philosophie de la conscience, il existe aussi une philosophie de la vie. D'ailleurs, tout le problème des rapports entre l'explication scientifique et la comprébension philosophique, entre sciences naturelles (Naturwissenschaften) et sciences humains (Geisteswissenchaften), qui est si central dans la phénoménologie, a été d'abord posé d'une façon systématique par Dilthey dans le contexte d'une Lebensphilosophie. La vie, c'est quelque chose qui ne peut pas être expliquée objectivement, c'est quelque chose qui doit être compris intérieurement, par un sujet lui-même vivant. Ce n'est pas par une analyse objective, mais seulement par une sorte de sympathie vécue que nous pouvons comprendre ce qu'est la vie. Bergson, qui défendait une philosophie du sujet, défendait aussi ce point de vue. La vie et la notion de "purposeful behavior», est-ce donc un cas où l'explication scientifique et objectiviste se retrouve déficitaire et doit être dépassée vers un mode de compréhension pbilosophique?

C'est là la vieille querelle entre mécanisme et vitalisme en BIOLOGIE. Et il me semble qu'ici aussi la science positive a effectivement évolué dans un sens qui rend caduque cette antinomie. C'est-à-dire que la science biologique, en transformant son mécanisme traditionnel, s'est trouvée en état d'expliquer objectivement le phénomène de la vie sans qu'elle soit devenue pour autant vitaliste. Le «nouveau mode d'analyse» de la biologie moderne aurait dépassé les alternatives classiques de méca- 
nisme et de vitalisme, et ceci sans aucun abandon de l'objectivisme et sans aucune concession à la philosophie du sujet. C'est ce qui ressort des discussions au sujet du sens du comportement animal.

Dire que le comportement d'un organisme a un sens, c'est dire que ce comportement ne peut se comprendre uniquement dans les termes d'un mécanisme classique. On ne peut décrire adéquatement le phénomène vital sans tenir compte du fait que le comportement d'un animal est quelque chose de significatif et de téléologique. L'animal agit non pas par simple hasard et selon les seules lois de la nature inanimée, mais il cherche à réaliser un certain but qu'il se propose lui-même. Le comportement animal ne peut être compris si l'on ne fait que le décomposer «en une multitude de processus partiels, extérieurs les uns aux autres dans le temps comme dans l'espace. ${ }^{21}$ On doit lui reconnaitre un sens et une finalité. Nous revoilà donc devant le concept de sens, concept si central dans la phénoménologie. Voyons donc ce que nous en dit Merleau-Ponty.

Le préjugé fondamental dans l'analyse de Merleau-Ponty est qu'un sens ne peut pas exister dans la nature; la notion de sens se réfère essentiellement à la notion de conscience. Ainsi dans La Structure du comportement Merleau-Ponty, après avoir fait remarquer que la science elle-même en est venue au concept de forme ou de sens, conclut pour sa part que « l'objet de la biologie est impensable sans les unités de signification qu'une conscience y trouve et y voit s'y déployer. ${ }^{22}$ Sa réflexion sur la science est donc de nature nettement transcendentale: le comportement animal ne peut se comprendre sans la notion de sens, et la notion de sens renvoie à la notion de conscience. Seulement, pour Merleau-Ponty, cette conscience qui «constitue 》 le sens organique, «pour qui » ce sens existe, n'est pas l'ego transcendental, mais c'est tout de même un sujet, le sujet percevant. Ainsi, pour Merleau-Ponty, les recherches de la science ont pour but essen-

21. MERLEAU-PONTY, La structure du comportement, Paris, P.U.F., 1963, p. 6.

22. Ibid., p. 173 . 
tiel l'exigence d'une nouvelle sorte de philosopbie; la leçon qu'il en tire c'est qu'il faut «définir à nouveau la philosophie transcendentale $\gg^{23}$ Cette philosophie transcendentale constituerait donc la vérité des visées scientifiques.

L'important maintenant, ce serait de comparer et de contraster les conclusions philosophiques de Merleau-Ponty au sujet du sens du comportement animal avec les analyses scientifiques des scientifiques eux-mêmes. On verrait peut-être par là que la science positive peut très bien incorporer la notion de sens dans ces analyses sans que celles-ci cessent d'être purement objectives. Faute de pouvoir ici examiner cette question en détail, je me contenterai de signaler certaines remarques de Wiener, Monod et Jacob.

D'abord, dans son article déjà signalé, Wiener dit que «the category of purpose is one of the fundamental categories in science. ${ }^{24}$ Il veut donc réhabiliter la notion de «teleological purposeful behavior» comme mode d'explication proprement scientifique. Mais la notion de «purpose » ou de «finalité » est pour Wiener strictement objective; elle désigne un comportement et ne se réfère pas à des mobiles conscients ou subjectifs. D'ailleurs pour Wiener rien n'empêche qu'on applique la notion de «purpose» à certains systèmes non-vitaux, ce qui implique que la vie elle-même n'a rien de spécifique, mais est seulement un certain genre de mécanisme. Il écrit :

23. Ibid., p. 241. J'ai examiné en détail la position de Merleau-Ponty à ce sujet dans mon étude, La Pbénoménologie de Merleau-Ponty, une recherche des limites de la conscience (Paris, Editions Klincksieck, 1973); voir ch. I, «Les rapports entre «structure» et «sens 》 et le problème de la conscience perceptive $»$, p. 35-40. On peut aussi remarquer que la position de Merleau-Ponty ne semble pas avoir changé au cours de sa carrière. Ainsi dans ses Résumés de cours faits au Collège de France (Paris, Gallimard, 1968), il écrit (p. 136, 137): \&Il est apparu que toute zoologie suppose de notre part une Einfüblung méthodique du comportement animal, avec participation de l'animal à notre vie perceptive et participation de notre vie perceptive à l'animalité ». «...l'ontologie de la vie, comme celle de la «nature physique», ne sort d'embarras qu'en tecourant, hors de tout artificialisme, à l'être brut tel qu'il nous est dévoilé par notre contact pcrceptif avec le monde. Ce n'est que dans le monde perçu qu'on peut comprendre que toute corporéité soit déjà symbolisme ».

24. V. WIENER, art. cit., p. 321. 
If the term purpose is to have any significance in science, it must be recognizable from the nature of the act, not from the study of or from any speculation on the structure and nature of the acting objet. This remark is important because it eliminates any incongruity in the application of the notion to nonliving machines. In other words, if the notion of purpose is applicable to living organisms, it is also applicable to non-living entities when they show the same observable traits of behavior. ${ }^{25}$

Comme c'est le cas avec la notion de causalité, la notion de «purpose », soutient Wiener, n'a vraiment de sens que dans la science moderne, dans un contexte non plus absolutiste mais probabiliste. Il existe une analyse rigoureuse et objective de «purposeful behavior » qui trouve son expression dans une théorie mathématique de la prédiction.

Les vues de Jacob et de Monod sont du même genre. Tous les deux insistent sur l'importance de la notion de téléologie pour la compréhension de la vie, (Monod préfère le terme «téléonomie », moins philosophique d'apparence). La propriété fondamentale des êtres vivants, dit Monod, est « d'être des objets doués d'un projet ». Cette notion, dit-il, est «indispensable... à la définition des êtres vivants. ${ }^{26}$ De même, Jacob dit « qu'on ne peut plus faire de biologie sans se reférer constamment au «projet» des organismes, au «sens ${ } . .{ }^{27}$ Et il fait remarquer combien cette nouvelle attitude diffère du réductionisme traditionnel. «Aujourd'hui au contraire, on ne peut plus dissocier la structure de sa signification.»

Seulement, pour Jacob et Monod, ces notions de «sens» «signification», «projet», 《téléologie 》, etc., ne se refèrent pas essentiellement à un sujet humain. A la base de ce phénomène de sens et de finalité, il n'y a pas pour eux une conscience constituante ou perceptive: «ce qu'a démontré la biologie, c'est qu'il n'existe pas d'entité métaphysique pour se cacher derrière le mot de vie. ${ }^{28}$ Il n'y a au fond qu'un mécanisme génétique qui

25. Ibid., p. 323.

26. J. MONOD, Le basard et la nécessité, Paris, Editions du Seuil, 1970, p. 22.

27. F. JACOB, La logique du vivant, Paris, Gallimard, 1970, p. 321.

28. Ibid., p. 327. 
obéit à une logique aveugle et tout à fait inconsciente. Jacob écrit :

L'organisme devient ainsi la réalisation d'un programme prescrit par l'hérédité. A l'intention d'une Psyché s'est substituée la traduction d'un message. L'être vivant représente bien l'exécution d'un dessein, mais qu'aucune intelligence n'a conçu. Il tend vers un but, mais quaucune volonté n'a choisi. Ce but, c'est de préparer un programme identique pour la génération suivante. C'est de se reproduite. ${ }^{29}$

Ce qui régit le comportement organique et est responsable pour son «sens», c'est un message génétique «écrit au hasard» (Monod). Comme le dit Monod :

L'ultima ratio de toutes les structures et performances téléonomiques des êtres vivants est donc enfermée dans les séquences de radicaux des fibres polypeptidiques... En un sens, très réel, c'est à ce niveau d'organisation chimique que gît, s'il y en a un, le secret de la vie. Et saurait-on non seulement décrire ces séquences, mais dénoncer la loi d'assemblage à laquelle elles obéissent, on pourrait dire que le secret est percé, l'ultima ratio découverte. ${ }^{30}$

Voilà donc dans le cas de la biologie moléculaire le mécanisme et l'objectivisme pleinement réhabilités selon un nouveau modèle, le modèle cybernétique. Monod parle même d'une «cybernétique microscopique $\gg{ }^{31} \mathrm{Et}$ Jacob dit qu' « Avec le concept de programme appliqué à l'hérédité, disparaissent certaines des contradictions que la biologie avait résumées par une série d'oppositions : finalité et mécanisme, nécessité et contingence, stabilité et variation. ${ }^{32} \mathrm{Si}$ aujourd'hui le concept de téléologie a de nouveau un sens dans la biologie, c'est grâce au concept de programme. ${ }^{33}$ La biologie moléculaire nous présente donc un cas de dépassement des alternatives classiques, un dépassement qui, jus-

29. Ibid., p. 10.

30. MONOD, op. cit., p. 110.

31. Ibid., p. 93.

32. JACOB, op. cit., p. 10.

33. Ibid., p. 17. 
tement, va dans le sens d'un mécanisme structuraliste ou cybernétique. ${ }^{34}$

La biologie moderne a l'ambition d'interpréter les propriétés de l'organisme par la structure des molécules qui le constituent. En ce sens, elle correspond à un nouvel âge du mécanisme. Le programme représente un modèle emprunté aux calculatrices électroniques. Il assimile le matétiel génétique d'un oeuf à la bande magnétique d'un ordinateur. ${ }^{35}$

Le cybernéticien $\mathrm{P}$. Idatte, pour sa part, maintient que «le pro. blème de la vie et le problème cybernétique ne font qu'un. ${ }^{36}$

Ainsi donc l'organisme n'est qu'une sorte de macbine, et le comprendre c'est dégager les algorithmes qui expriment les interactions entre ses parties constituantes. Aucun appel donc à un mode de compréhension non objectiviste. ${ }^{37}$ Wiener, Monod et Jacob conçoivent l'organisme selon le modèle d'une machine. Et L.-J. Delpech, parlant de cette «imitation \% cybernétique de la vie intelligente qu'est l'homéostat d'Ashby et d'autres appareils mécaniques de ce genre qui manifestent toutes les propriétés des êtres vivants et intelligents, peut conclure:

34. Foucault n'a pas manqué de percevoir les implications «structuralistes de la biologie moléculaire. Voir son compte rendu du livie de Jacob dans Le Monde, en décembre 1970. Voir aussi le compte rendu du livre de Monod par F. Dagognet dans le même numéro.

35. JACOB, op. cit., p. 17.

36. P. IDATTE, Clefs pour la cybernétique, Paris, Eds. Seghers, 1969, p. 108. Cf. aussi K. Sayre, "Philosophy and Cybernetics» in Pbilosopby and Cybernetics, New York, Simon and Schuster, 1967, p. 13: "The unity of cybernetics from the beginning, therefore, rested upon a dismissal of what appears different between living and nonliving systems .

37. «Vitalism has won to the extent that even mechanisms correspond to the time-structure of vitalism; but as we have said, this victory is a complete defeat, for from every point of view which has the slightest relation to morality or religion, the new mechanics is fully as mechanistic as the old. Whether we should call new point of view materialistic is largely a question of words: the ascendance of matter characterizes a phase of nineteenth-century physics far more than the present age, and \& materialism 》 has come to be but little more than a loose synonym for «mechanism». In fact the whole mechanist-vitalist controversy has been relageted to the limbo of badly posed questions 》. N. WEINER, Cybernetics, p. 44. 
le fait primordial est l'existence - désormais établie - de solutions mécanistes du problème que pose l'explication des comportements considérés jusqu'ici comme l'apanage de la matière vivante.

Des concepts tel que ceux d'adaptation, d'apprentissage, de finalité, etc., relativement bien précisés sur le plan de la psychologie, sont restés très obscurs du point de vue physiologique. Il semble qu'enfin les voies d'approche soient trouvées qui, en permettant d'étudier rationnellement ces phénomènes, vont conduire à des résultats substantiels et objectifs. ${ }^{38}$

Et finalement P. Idatte insiste : «Nous ne saurions trop souligner la matérialité fondamentale des opérations et des systèmes cybernétiques. ${ }^{39}$

Quoi qu'on pense du matérialisme comme position philosophique, on peut toujours se demander si ce matérialisme "opératoire » de la science n'est peut-être pas absolument indispensable au projet scientifique, une hypothèse de travail aussi nécessaire que son postulat d'objectivité.

(4) Comme on a pu le voir, la biologie a renouvelé son appareil conceptuel et ainsi dépassé les alternatives classiques de mécanisme et de vitalisme grâce à des modèles cybernétiques. Ce qui régit le comportement d'un organisme, c'est en fin de compte le «message », $l$ ' « information », le «code» génétique. Ainsi on en vient à la CYBERNÉTIQUE, qui semble bien occuper la place centrale dans l'épistémologie de la science actuelle. Une philosophie de la science et une critique phénoménologique de la science seraient bien obligées tôt ou tard de tenir compte de cette nouvelle science pluridisciplinaire, cette «science-carrefour». Ici comme ailleurs, je me bornerai cependant à quelques brèves remarques, faute de pouvoir traiter le sujet d'une manière plus adéquate.

Dans la cybernétique la notion-clef de la phénoménologie, la notion de signification, joue aussi un rôle de première impor-

38. L.-J. DELPECH, La Cybernétique et ses théoriciens, Paris, Casterman, 1972 , p. 69.

39. P. IDATTE, ibid., p. 18. 
tance. Mais ici la signification devient l'information, et ce concept est expliqué en termes uniquement matériels (dans le concept d'information, il est uniquement question de l'organisation et l'échange de signaux matériels). Cette notion d'information n'a pas d'équivalent dans la science classique, et elle exprime un dépassement de l'antinomie ancienne entre nature et conscience. Si d'ailleurs Merleau-Ponty s'est donné le problème de «comprendre les rapports de la conscience et de la nature ${ }^{40}$ et a cru résoudre ce problème de rapports dans le sens d'une conscience perceptive, d'autres l'ont tenté aussi, mais l'ont résolu dans le sens d'une théorie de l'information. Le concept d'information est un concept purement objectif ; l'information tst ce qui peut être mesuré objectivement, ce n'est pas ce que détient nécessairement une conscience; ce concept n'implique pas celui de conscience. ${ }^{41}$ Mais, comme partout ailleurs dans la science opérationnelle moderne, le concept d'information ne se refère pas non plus à une nature en soi, à la «nature» par opposition à l'《esprit ». C'est justement cette dichotomie entre nature et esprit qui se trouve ici dépassée. Comme le dit Wiener : «Information is information, not matter or energy. No materialism which does not admit this can survive at the present day. ${ }^{42}$ Implicite dans cette affirmation est l'idée que la notion d'information exige l'abandon, non pas du matérialisme tout court, mais seulement du matérialisme classique. Le concept d'information désigne plutôt l'organisation de la matière; c'est un concept opérationnel et structural. ${ }^{43} \mathrm{Le}$

40. La structure du comportement, p. 1.

41. «The concept of information in the technical sense provides a purely quantitative measure of communication transactions which abstracts entirely from the interests and meanings of the agents involved $» . K$. SAYRE, art cit., p. 7. "An information source is purely ard simply any device (man, machine, or other) whose output symbols are not perfectly predictable in advance». J.L. MASSEY, «Information, Machines and Men, 》 in Pbilosophy and Cybernetics, p. 56-57.

42. Cybernetics, p. 132.

43. Le concept d'information «définit unc grandeur physique liée, non pas à l'aspect matériel ou énergétique du système, mais à son aspect d'organisation ». F. MEYER «Situation épistémologique de la biologie», in Logique et connaissance scientifique, Paris, Gallimard, Encyclopédie de la Pléiade, 1967, p. 804. «... information is a measure, in appropriate units, of certain statistical characteristics. $\gg \mathrm{K}$. SAYRE, Consciousness : A Pbilosopbic Study of Minds and Macbines, New York, Random. House, 1969, p. 179. 
contraire de l'information est le bruit, c'est-à-dire la désorganisation d'une "matière» (chimique, sonore, électrique, etc.). Ainsi le lieu où réside l'information n'est pas obligatoirement une conscience. Au contraire, comme c'est le cas pour la vie dans la biologie, la conscience elle-même est réinterprétée en termes de mécanisme structural ; elle est conçue cybernétiquement. ${ }^{44} \mathrm{La}$ conscience est elle-même objectivée; la logique des opérations conscientes n'est pas elle-même consciente; le calculus ratiocinator devient machina ratiocinatrix. ${ }^{45}$ Comme l'observe Wiener : "It is not in the least surprising that the same intellectual impulse which has led to the development of mathematical logic has at the same time led to the ideal or actual mechanization of processes of thought. ${ }^{46}$ D'autres théoriciens de la cybernétique sont sur ce point d'accord avec Wiener : « it seems inevitable that a more precise and articulate conception of what is involved in the intelligent behavior of human beings will result in an increased ability to program this behavior into a sufficiently capacious digital computer. ${ }^{47}$ Du moment en effet où la science arrive à transformer en problèmes ce qui avait été auparavant pris pour des mystères, tels l'intelligence et la conscience humaines, du mo-

44. Il y aurait lieu d'examiner dans le détail la position défendue par $\mathrm{K}$. Sayre dans son livre provocant, Consciousness... (cf. référence dans note précédente). L'originalité de Sayre réside dans sa tentative de défendre une interprétation mécaniste et matérialiste de la conscience, renouvelée selon des modèles cybernétiques. «My approach, dit-il, will be to formulate an entirely quantitative model of the conccious response in terms of the basic concepts of information theory $\gg$. (p. 152)

45. «Il faudra par conséquent nous défaire de cette idée d'un homme porteur, donneur ou créateur de sens pour nous contenter de constater que l'homme manipule des signes et est lui-même possédé par des champs sémiologiques selon des fonctionnements dont les combinaisons se réduisent, en dernière analyse, à des combinaisons d'atomes dont le jeu appartient à celui des forces naturelles». J. BRUN, «La linguistique et la philosophie», Contrepoint, no. 4. été 1971 , p. 26. Voir aussi L. COUFFIGNAL, La cybernétique, Paris, P.U.F., «Que sais-je? », 1968, p. 109: «Les machines à calculer fonctionnant en machines à raisonner peuvent, de nos jours, effectuer automatiquement toutes les opérations logiques qu'effectue l'esprit humain, notamment construire une théorie déductive: il faut dès lors admettre que le raisonnement déductif, par exemple la construction des mathématiques, n'exige pas la mise en oeuvre d'une «faculté de l'esprit», par exemple celle qu'on l'on appelle «intelligence».

46. Cybernetics, p. 12.

47. F. CROSSON \& K. SAYRE, Pbilosopby and Cybernetics, p. xii. 
ment ò̀ elle réussit à traduire ces phénomènes en langage scientifique, il n'y a plus aucune raison pour qu'elle ne puisse pas aussi expliquer ou résoudre ces problèmes d'une façon purement empirique ou scientifique. L'essentiel réside tout entier dans cette traduction, non pas dans la résolution du problème, qui suit nécessairement. Ce serait une autre question que de savoir si dans cette traduction quelque chose d'essentiel au phénomène n'est pas tout simplement oublié.

Si donc on utilise en cybernétique des termes employés aussi par une philosophie phénoménologique - téléologie, sens, conscience, autonomie, etc. - ce n'est pas que la science soit en train de quitter son matérialisme, c'est, au contraire, qu'elle essaie de «naturaliser» ces concepts. Dans la cybernétique on arrive même à «expliquer» - c'est-à-dire à cissoudre dans une explication empirique - la liberté et le libre-arbitre. C'est un fait empiriquement observable que ce servo-mécanisme qu'est l'homme se distingue d'autres mécanismes en ceci qu'il se comporte comme s'il n'était pas contraint par son environnement ni par ses propres impulsions ou signaux internes. 'Tout ce qu'il faut pour expliquer ce phénomène cybernétiquement est de reconnaître dans le système à retour un délai entre l'apparition du signal d'erreur et la décision. Le signal d'erreur n'agir pas directement; il peut être lui-même analysé et même ignoré en fonction d'autres informations dont dispose le système. Ce n'est donc pas de l'«anthropomorphisme» que de parler de sens et de finalité etc. au sujet des êtres non-humains, car l'homme a été lui-même objectifié, naturalisé. ${ }^{48}$ Écoutons à ce sujet Wiener :

We also wish to explain why we use the humanistic terms purpose and teleology in the description of the behavior of some machines. The question of whether machines are or can be like men or the higher animals does not guide our choice. This question is on the main irrelevant for scientific objectives. We believe that men and other animals are like machines from the

48. «L'homme se trouve ainsi dessous, la culture n'est plus que ce gui s'oppose à la nature pour la refaire selon des rythmes qui ne sont pas les siens, mais bien ce qui fait partie de cette nature elle-même. (...) L'homme se trouve donc réintégré dans la nature d'une façon si totale qu'on peut se demander en quoi il se distingue de l'amibe ou de la pierre qui n'ont ni histoire ni culture». J. BRUN, art. cit., p. 30. 
scientific standpoint because we believe that the only fruitful methods for the study of human and animal behavior are the methods applicable to the behavior of mechanical objects as well. Thus, our main reason for selecting the terms in question was to emphasize that, as objects of scientific enquiry, humans do not differ from machines. ${ }^{49}$

Ce qui est intéressant dans ce texte, c'est l'approche de l'auteur. En effet, Wiener n'est pas en train d'énoncer une doctrine métaphysique, à savoir que l'homme est une machine; ce n'est pas du matérialisme classique, philosophique. Wiener ne parle pas en philosophe mais en scientifique; c'est pour des raisons de méthode que la science considère l'homme comme s'il n'était rien d'autre qu'une machine. Cette distinction est très importante et, bien comprise, elle est de nature à déjouer beaucoup de critiques philosophiques. Le matérialisme operatoire de la science est comme une hypothèse de travail, une hypothèse de travail qui est, je suis porté à le croire, la condition même d'une analyse scientifique. Par conséquent, s'attaquer comme le font beaucoup de phénoménologues au matérialisme de la science, à son objectivisme et à son naturalisme, c'est tout simplement s'attaquer à la science elle-même. Et une telle attaque n'aurait sans doute aucune chance de succès. Ce serait même de l'irrationalisme pur et simple.

La conclusion, en ce qui concerne la nature actuelle du projet scientifique, me semble donc s'imposer. Il y a eu effectivement dans la science moderne un bouleversement spectaculaire de catégories et de concepts. Seulement, je crois que ce changement épistémique devrait être considéré comme une évolution plutôt que comme une révolution. Car tous les développements sont des développements à l'intérieur du mécanisme qui se trouve par là non abandonné mais modifié. Comme Wiener le dit lui-même: "the new mechanics is fully as mechanistic as the

49. WIENER, art. cit., p. 326. 
old ${ }^{50}$. Et nous avons vu Jacob parler d' «un nouvel âge de mécanisme ». Là où il faut chercher la nouveauté de la science moderne, c'est dans l'usage qu'elle en fait des modèles statistiques et probabilistes. Le développement d'une logique ou d'une mathématique des probabilités et de la «prédiction» a par là permis à la science d'étendre ses analyses mécanistes à des domaines qui, jusque là, résistaient à ce genre de traitement mécaniste. C'est ainsi que le vitalisme se trouve sans doute définitivement évincé comme option scientifiquement valable. La cybernétique elle-même se considère comme une branche de la mécanique scientifique ${ }^{51}$.

Husserl n'avait donc pas tort quand il croyait discerner une «crise des fondements» dans les sciences. Ni Merleau-Ponty quand il croyait avoir découvert dans les recherches scientifiques une tentative de dépasser les anciennes alternatives. Seulement, l'évolution effective de la science ne l'amenait pas vers une réconciliation avec la phénoménologie. Si la science a reconnu l'importance des notions de sens, finalité, expérience, etc., c'est parce qu'elle a trouvé dans une épistémologie nouvelle le moyen de rendre compte de ces notions objectivement. La situation actuelle semble donc être la suivante: ou bien la phénoménologie, en perdant toute exclusivité sur ses concepts fondamentaux, a tout simplement perdu la partie et n'a plus aucune raison d'exister, devant être remplacée par une philosophie qui prend la connaissance scientifique comme modèle épistémologique, tel le positivisme ou le structuralisme, ou bien il lui reste un travail à faire, mais dans ce cas-ci elle ne trouvera aucun secours du côté d'une science devenue plus positiviste que jamais.

Les choses étant ainsi, il convient de s'interroger sur les rapports possibles entre une philosophie du type phénoménologique (débarrassée de ses illusions d'utilité scientifique) et la science (mécaniste et positiviste). Il devient même absolument indispensable qu'on repose la question d'une façon plus radicale que jamais.

50. Cybernetics, p. 44.

51. Ibid., p. 10. 
Je suis tout à fait d'accord avec Paul Ricoeur quand il estime qu'on ne doit pas vouloir trop vite intégrer les résultats des sciences particulières à des positions philosophiques, mais qu'il faut plutôt laisser ces disciplines aller « jusqu'au bout des exigences qui [ les] définissent ${ }^{52}$. Car il faut admettre que les philosophes ont été souvent assez imprudents dans leurs tentatives de dégager une "philosophie implicite» dans la science et, sous prétexte que le scientifique est philosophe à son insu, d'entrer d'emblée dans le débat sur la signification de certaines notions en science. L'exemple classique de ce genre d'ingérence dans les affaires intérieures de la science est la fameuse polémique de Bergson avec Einstein. Le résultat désastreux de cette aventure devrait enseigner aux philosophes plus de prudence. Mais, au sujet des rapports entre science et philosophie, on continue de trouver dans la littérature phénoménologique certaines équivoques importantes qu'il importe de dissiper. Je n'en mentionnerai que trois.

(1) La première équivoque dans cette question des rapports entre philosophie et science concerne les notions de fait et d'interprétation. Il y a une erreur courante qui consiste à dire que la science ne découvre et ne parle que de simples faits. Selon ce point de vue, la responsabilité de la philosophie serait d'interpréter correctement ces faits. C'est la position de Sartre dans son livre sur les émotions. Pour Sartre, le scientifique n'est qu'un «collectionneur» de faits, et la science n'est «qu'une somme de faits hétéroclites dont la plupart n'ont aucun lien entre eux ${ }^{\mathbf{5 3}}$. Je crois qu'il n'est nullement besoin de critiquer ici cette conception de la science, son absurdité étant pleinement évidente.

52. P. RICOEUR, «La philosophie à l'âge des sciences humaines》, Cabiers de pbilosopbie (publié par le groupe d'études de philozophie de l'Univcrsité de Paris), no. 1, janvier 1966, p. 99. Pour une analyse de la position de Ricoeur vis-à-vis de la pensée objective, voir mon article « Ricoeur et la non-philosophie », Luval théologique et philosophique», vol. XXIX, no 3, oct. 1973, p. 227-241.

53. J.-P. SARTRE, Esquisse d'une théorie des émotions, Paris, Hermann, 1965 , p. 9. 
Il n'existe pas de purs «faits», et le scientifique ne fait pas que les «découvrir» comme Christophe Colomb découvrait l'Amérique. Au contraire, tout fait scientifique est inséparable d'un certain schéma interprétatif et n'a de sens et d'existence comme fait que par rapport à lui. La division du travail que Sartre voudrait établir entre science et philosophie est donc à rejeter au départ.

La position de Merleau-Ponty à ce sujet est plus nuancée, mais tout aussi équivoque. Merleau-Ponty reconnaît que la logique d'induction classique n'est pas la logique réelle de la démarche scientifique - ce que Sartre semble ignorer - et que, dans la pratique, le scientifique peut ne pas interpréter et «comprendre $»$ les faits qu'il note. L'erreur de Merleau-Ponty est de conclure que, ceci étant, le scientifique fait lui-même de la «philosophie». Il dit, parlant du sociologue : «Au moment de l'interprétation, il est lui-même déjà philosophe ". Ainsi son erreur est au fond la même que chez Sartre: il ne peut attribuer un rôle philosophique au scientifique (et sur cette base justifier une collaboration entre philosophe et science), que parce qu'il a su au départ implicitement séparé description et interprétation et a attribué le rôle d'interprétation ou de compréhension à la philosophie.

Mais si l'explication scientifique est, de par sa propre nature, solidaire d'une certaine interprétation, le scientifique dans son travail d'interprétation des faits ne fait pas de la philosophie, et ce n'est pas à cet égard qu'on découvrirait des rapports entre philosophie et science. Il faudrait plutôt reconnaître que la science est une compréhension autonome, qu'elle constitue un univers de discours à elle et que celui-ci en dernière analyse se fonde et se justifie lui-même. Ainsi on doit prendre note avec MerleauPonty quand il dit que «le philosophe professionnel n'est pas disqualifié pour réinterpréter des faits qu'il n'a pas lui-même observés, si ces faits disent autre chose et plus que le savant y a vu», car ce que «veut dire » un fait scientifique n'est justement pas séparable du contexte interprétatif de la science en question; le fait n'est ce qu'il est et ne signifie ce qu'il signifie que parce qu'il a été au préalable filtré à travers un certain schéma interprétatif de la science. 
(2) La deuxième équivoque est liée à la première; elle réside dans cette idée que la science est ou contient une pbilosopbie implicite. Cette argument a été souvent utilisé par les philosophes pour justifier leur ingérence dans la science, car si le scientifique travaille avec certaines présuppositions proprement philosophiques, le phénoménologue peut lui montrer que ces présuppositions sont ou ne sont pas de nature à rendre pleine justice à ses découvertes. C'est le sens des objections de Merleau-Ponty à la Gestaltpsychologie. J'estime cependant pour ma part que, dans le meilleur des cas, ce rôle de la philosophie à l'égard de la science serait plutôt négatif, en ce sens que le philosophe peut parfois signaler au scientifique l'existence dans sa pensée de certaines idées proprement philosophiques et qui sont, comme telles, nuisibles à la démarche scientifique. Mais dans la mesure où la science devient plus «scientifique», elle assume elle-même la responsabilité de ses concepts de base, et ces concepts n'ont plus aucune autre source que la praxis scientifique elle-même. Le seul critère pour juger de la justesse d'un concept scientifique devient alors l'utilité de ce concept comme postulat de travail, et elle n'a plus alors rien de proprement philosophique. Tel est justement le sens du mécanisme de la science contemporaine.

$\mathrm{Au}$ fond, ce qu'il faut récuser ici, me semble-t-il, c'est la conception hiérarchique de la connaissance, réliquat de la scolastique, où la science est au rez-de-chaussée et la philosohie occupe l'étage supérieur, conception qui accorde le rôle de juge au philosophe qui est seul habilité à «comprendre » l'ultime signification des notions scientifiques et qui leur accorde ou refuse son imprimatur. Ce qu'il faut récuser donc, c'est une conception linéaire ou unidimensionnelle de la connaissance et de l'intelligence humaine, conception, il faut le noter, que les philosophes positivistes partagent aussi.

En somme, je dirais qu'il n'y a pas, en principe, de philosophie dans la science, mais que - et ceci est déjà un tout autre problème - l'existence de la science pose pour la philosophie des problèmes proprement philosophiques. L'exemple qui est peutêtre le plus éclatant est l'interprétation de l'homme qui nous est proposée par la cybernétique. Pour cette science l'homme 
n'est qu'un servo-mécanisme; il est compris uniquement selon le modèle du système à retour (feed-back mechanism) à traiter l'information (chose, elle aussi, matérielle). C'est donc dans la cybernétique qu'est accomplie la revanche du corps-machine sur l'esprit cartésien qui est lui-même finalement matérialisé. Ce matérialisme complet est, nous l'avons vu, un matérialisme opératoire ou fonctionnel et non pas philosophique ou dogmatique. Mais même si ce matérialisme scientifique est méthodologiquement irréprochable, il n'est pas sans poser de graves problèmes pour la philosophie. Car la philosophie aurait alors à décider si, dans la réalité, l'homme n'est vraiment rien de plus qu'une machine à calculer et si, au matérialisme scientifique, devrait correspondre un matérialisme philosophique.

(3) Enfin, la troisième équivoque à dissiper concerne la question des limites de la science et la possibilité de dialogue entre philosopbie et science. La science a-t-elle des limites, est-elle essentiellement limitée? Ce serait à mon sens une grave erreur que de concevoir cette question de limites dans le sens d'un partage $d u$ réel entre science et philosophie. C'était là l'erreur du vitalisme qui demandait l'impossible: la reconnaissance ou la vérification par la science d'un phénomène - la vie - qui était censé ne pouvoir être expliqué scientifiquement. Demande impossible, car si un phénomène peut être observé scientifiquement, il est sans doute nécessaire que, tôt ou tard, il pourra aussi recevoir une explication en termes purement scientifiques. Ainsi on ne peut pas limiter la science à certains phénomènes seulement en réservant d'autres pour un autre mode de compréhension. Le projet même de la science l'oblige à essayer de tout expliquer objectivement. C'est dire que l'analyse scientifique est essentiellement totalitaire. Il serait tout simplement anti-scientifique pour la science de renoncer à expliquer quoi que ce soit - du moment qu'il existe et est constaté empiriquement - d'une manière scientifique, purement objective.

Ce serait donc une équivoque de soutenir que la philosophie et la science peuvent entrer en dialogue si seulement la science «reconnaît ses limites». En dépit de l'esprit oecuménique qui est de bon ton de nos jours, il faut tout de même reconnaître qu'il y a des cas où le dialogue n'est ni souhaitable 
ni même possible. Il se peut qu'il y ait quelque chose qui ne peut pas être saisi et compris au niveau de la science, mais si tel est le cas - et je suis personnellement convaincu qu'il en est ainsi - il s'ensuit nécessairement que le fait que ce quelque chose ne peut être exprimé scientifiquement est lui-même scientifiquement inexprimable. Si la science a des limites, ces limites ne peuvent être connues au niveau de la science. Car à ce niveaur, quand on fait de la science, la science apparaît comme autonome, totalitaire, fermée sur elle-même et illimitée intérieurement. Ainsi je ne vois aucun sens à parler de dialogue entre philosophie et science, de collaboration et de compréhension mutuelles. L'équivoque ici consiste à confondre le dialogue entre science et philosophie, qui est impossible, avec un dialogue entre scientifique et philosophe, dialogue qu, lui, est bien possible, mais à la condition que le scientifique ne parle pas seulement en tant que scientifique, à condition donc qu'il sorte de l'univers du discours scientifique ${ }^{54}$

Mais si, à l'encontre de ce que pensait Merleau-Ponty, il ne reste à la philosophie phénoménologique aucun rôle positif à jouer dans l'élaboration d'une connaissance positive du monde, de la vie et même de l'homme lui-même, ne perd-elle pas simplement toute raison d'être? S'il n'y a pas de place dans l'univers du discours scientifique pour un langage philosophique, réflexif, quelle peut bien être la justification de ce dernier? C'est la réponse à cette question que j'aimerais seulement suggérer pour finir.

\section{IV}

Ce qui finalement est peut-être le plus déconcertant chez Merleau-Ponty, chez Sartre et chez d'autres phénoménologues, c'est la curieuse habitude qu'ils ont de faire appel à des faits

54. Je dois donc me censurer moi-même si, dans ma communication au XVe congrès de l'A.S.P.L.F., «Langage scientifique et langage philosophique », La Communication (Montréal, Eds. Montmorency, 1971), jai pu donner l'impression, par une tournure de phrase sans doute malheureuse, que je croyais à la possibilitć d'un dialogue entre pbilosophie et science, en tant que disciplines. 
empiriques, mis en lumière par la science, afin finalement d'en tirer des conclusions d'un tout autre ordre que scientifique, des conclusions transcendentales ou réflexives quant à la signification philosophique de ces faits. La Phénoménologie de la perception constitue un bon exemple de ce genre de pratique. A plusieurs occasions, Merleau-Ponty résume des découvertes et expériences des psychologues et des physiologistes et puis, brusquement, il change de vitesses pour ainsi dire et commence à parler un tout autre langage. C'est peut-être ce mélange de discours que Piaget avait en vue quand il disait que sa lecture du livre de MerleauPonty lui produisait une impression «ahurissante ${ }^{55}$. Et c'est justement cette confusion de langage, d'ordres de discours, qu'il me semble absolument nécessaire d'éviter si l'on ne veut pas réduire en fin de compte la science à la philosophie ou la philosophie à la science. Or il n'y a sans doute pas grand danger à l'heure actuelle de voir la science réduite à la philosophie, mais, par contre, il y a grand danger que la philosophie phénoménologique, en se basant sur des données scientifiques qu'elle n'a pas elle-même établies et dont elle ne peut pas rendre compte, perde toute raison d'être (car la meilleure explication des données scientifiques viendra du côté de la science). On ne peut réconcilier le matérialisme et le mécanisme scientifiques avec la priorité mise sur la conscience par la phénoménologie, ni avec ses analyses intentionnelles et acausales, car ces deux positions sont tout simplement antithétiques et mutuellement exclusives, aussi longtemps qu'on essaie de les faire participer à un même univers du discours.

Ainsi, si l'on se situe dans une philosophie du sujet, l'important, il me semble, est de savoir de quoi on parle et de quoi on ne parle pas. C'est dire qu'il faut renoncer à une conception linéaire et hiérarchique de la connaissance, renoncer à joindre le point de vue objectif et le point de vue réflexif - pour employer les termes de Merleau-Ponty. Il faut savoir opérer une distinction absolument fondamentale et de première importance, la distinction entre analyse scientifique (empirique) et analyse philosophique (transcendentale), et il faut ne pas confondre les

55. J. PIAGET, Sagesse et illusions de la philosopbie, Paris, P.U.F., 1968, p. 36. 
deux dans une vaine tentative de réconciliation. Husserl lui-même, en dépit du fait qu'il insistait beaucoup sur l'utilité de la phénoménologie pour la psychologie, reconnaissait ceci quand il disait dans son Nachwort que «la phénoménologie transcendentale n'est en aucun sens une psychologie, même une psychologie phénoménologique ».

Afin justement de défendre la philosophie du sujet contre le réductionnisme positiviste, il serait donc indispensable de commencer par rendre à la science sa totale liberté et l'exclusivité quant à une connaissance positive du réel. Si, par exemple, on ne peut comprendre les organismes vitaux qu'en tenant compte du caractère téléologique et significatif de leur comportement, cela ne veut pas dire que la philosophie aurait un rôle positif à jouer dans l'explication de la vie et que la biologie devrait être «fondée» sur la philosophie; cela veut dire tout simplement que les concepts de «téléologie» et de «sens» doivent devenir des concepts positifs et objectifs. Et c'est justement ce qui est arrivé, comme j'ai essayé de le montrer. L'avance même de la science enlève à la philosophie son privilège spécial dans l'explication du réel.

Mais cette perte apparente est en fait un grand bénéfice. A mon sens, il ne peut être que salutaire à la philosophie du sujet d'être, enfin, débarrassée de connaissances objectives. Car dépouillée de tout empirisme, la philosophie serait amenée à découvrir que son véritable langage est d'un tout autre ordre qu'empirique. Le «sujet» de la phénoménologie et de la philosophie réflexive n'est pas un sujet (un objet) mondain, mais bien plutôt la condition de possibilité transcendentale du monde. La philosophie ne peut rien ajouter ni rien soustraire à la connaissance scientifique; elle ne peut que s'étonner devant ce produit de l'esprit humain et s'interroger sur les conditions de possibilité et sur la signification d'une telle activité.

Une véritable philosophie du sujet renoncera ainsi à prescrire des «limites» à la science. La science n'a pas de limites, si l'on entend par là qu'il existe une partie du réel qui échappe et résiste, par nécessité, à une analyse scientifique (c'était là, on l'a $\mathrm{vu}$, l'erreur du vitalisme). La science est, en principe, capable de tout expliquer - du point de vue scientifique. De même, la 
science est en fin de compte et en principe le seul juge de ses concepts opératoires, car le sens même de ces concepts est fonction de la praxis scientifique elle-même, ce qui veut dire qu'un concept en science est «vrai » s'il sert utilement à coordonner des données.

Le positivisme - et le structualisme - a donc raison quand il insiste sur l'autonomie de la science. Mais, ce qu'il faut remarquer également, c'est que, érigé en philosophie, le positivisme devient aussitôt absurde et intenable. Le propre d'une philosophie positiviste est de distinguer entre science et philosophie (métaphysique) et de dire que seule la première a valeur de connaissance tandis que la seconde ne peut être qu'une «sagesse »(Piaget), discours non rationnel mais émotif. Le structualisme pour sa part affirme que le seul savoir est la science et que tout le reste (dont la phénoménologie) n'est qu' «idéologie ». En somme pour le positivisme, la seule connaissance valable est la connaissance logico-empirique ${ }^{56}$. Mais ce point de vue est, comme je l'ai dit, absurde et intenable, de la même façon que l'est le scepticisme.

Le sceptisme pur est absurde, on le sait, parce qu'il se contredit lui-même dès qu'il s'érige en thèse: dire que rien n'est vrai ou certain, c'est déjà affirmer une vérité qui est censée être certaine, à savoir que rien n'est certain. C'est ce même genre de contradiction interne qu'on retrouve dans le positivisme. Dire qu'il n'y a qu'un mode de connaissance, la science, est déjà une affirmation non pas scientifique mais bien plutôt philosophique. C'est une affirmation méta-scientifique, une affirmation non de la science mais concernant la science. Ainsi, cette philosopbie qu'est le positivisme prétend que la science seule nous donne des vérités, mais c'est lui-même comme philosophie qui nous donne cette «vérité »! De même, il faudrait conclure, si l'on était structuraliste, que l'idée structuraliste que tout sauf la science est idéologie n'est elle-même qu'une affirmation vainement idéologique!

Mais comme il existe tout de même un scepticisme vécu, il existe aussi un positivisme vécu, et c'est la science. Le discours

56. Ainsi Monod parle de «la connaissance objective comme seule source de vérité authentique $\gg . O p$. cit., p. 185. 
scientifique est positiviste et structuraliste; il est en effet totalitaire et impérialiste dans la pratique et ne reconnaît implicitement comme valable d'autre discours que le discours objectif, positif. Mais dire ceci est en même temps indiquer ce qui constitue les véritables limites de la science. Puisque la science re peut admettre comme vrai que ce qui est conforme à ses exigences méthodiques, il y a dans la science, pour reprendre une expression de Merleau-Ponty, comme une sorte de cécité mentale qui l'empêche de ne voir quoi que ce soit autrement qu'objectivement. Ou comme Heidegger le dit : "La science n'atteint jamais que ce que son mode propre de représentation a admis d'avance comme objet possible pour lui ${ }^{57}$. Ce que la science ne peut pas voir, c'est justement parce qu'elle est science qu'elle ne peut pas le voir.

Mais si l'on peut même reconnaître et dire ce que nous venons de dire, si l'on peut parler rationnellement des limites de la science et de la vision scientifique, c'est justement parce que la science n'est pas le seul mode de connaissance ou de discours rationnel. L'exercice même de la critique philosophique démontre concrètement qu'il existe un autre mode de discours qui est à la fois rationnel et non-scientifique. On voit bien donc que la philosophie du sujet est possible, même aujourd'hui. Elle est possible et se justifie comme entreprise intellectuelle car elle sait que le sujet, la conscience, l'homme enfin peut se considérer lui-même objectivement, scientifiquement, alors que la science ignore tout de ce sujet qui la rend elle-même possible en se niant comme sujet. Parce qu'elle ne considère l'homme que comme un «special sort of machine ${ }^{58}$, la science est en effet la «mort de l'homme», comme dirait Foucault. Mais ce qu'il faut remarquer, c'est que cette science qui dissout l'homme dans la nature objective n'est pas elle-même autre chose qu'une activité et une création humaine. A ce moment, la question proprement philosophique et réflexive reçoit toute sa justification et toute son urgence : qui est donc cet étrange sujet qui a le pouvoir de s'oublier et de se nier lui-même dans la science, dans ce jeu

57. M. HEIDEGGER, Essais et conférences, Paris, Gallimard, 1958, p. 200.

58. WIENER, The Human Use of Human Beings, New York, Avon Books, 1967 , p. 107. 
intellectuel qu'il a lui-même inventé ? «Car enfin qu'est-ce que l'homme dans la nature? » (Pascal) Cette question que seule peut élucider une analyse non objective mais réflexive, non empirique mais transcendentale, cette question ne peut pas être esquivée du moment que le sujet qui se perd dans l'objectivisme se rend compte de ce qu'il est en train de faire. L'objectivisme, poussé à ses limites, peut réveiller le sujet transcendental qui s'ignorait. Et ainsi le philosophe est bien à cet égard tel que Merleau-Ponty le décrit: l'homme qui s'éveille et qui parle ${ }^{59}$.

Au terme de ces réflexions, il est donc devenu évident que la philosophie du sujet est non seulement possible, elle est même absolument nécessaire, car, sans elle- et c'est une chose que Merleau-Ponty a clairement vu —, "l'homme devient vraiment le manipulandum qu'il pense être, on entre dans un régime de culture où il n'y a plus ni vrai ni faux touchant l'homme et l'histoire, dans un sommeil ou un cauchemar dont rien ne saurait le réveiller ${ }^{60} \gg$. Sans la philosophie, c'est-à-dire sans vérité trans-scientifique, méta-physique, l'homme est en effet réduit à riêtre rien de plus qu'un servo-mécanisme, un robot mécanique; il est condamné à vivre dans un monde où il n'y a plus, à proprement parler, ni vérité ni liberté mais seulement l'efficacité. Or la science ne redeviendra sans doute jamais philosophie, et c'est justement ceci qui rend indispensable la philosophie. Comme philosophie du sujet, analyse réflexive, elle devrait être une connaissance non-scientifique, trans-empirique, méta-physique, une connaissance résolument bumaine. L'enjeu de toute cette question. des rapports entre philosophie et science, l'enjeu de la possibilité d'une philosophie du sujet et de la philosophie comme connaisance autonome et transempirique n'est, en dernière analyse, rien moins que l'humanité de l'homme. La science dit vrai:

59. Eloge de la pbilosophie, Paris, Gallimard, 1953, p. 100.

60. L'oeil et l'esprit, p. 12. Ma position diffère de celle de Merleau-Ponty en ceci qu'il croit que cette issue peut être évitée pourvu que la science "redevienne philosophie», comme il le dit, tandis que je considère que cette idée d'une jonction entre philosophie et science n'est pas raisonnable et, par conséquent, ja ne vois d'espoir que si l'on reconnaît, au-delà de la science, la possibilité d'un autre mode de connaissance qui serait la philosophie. 
l'homme n'est qu'une machine, mais cette vérité-là n'est pas la vérité dernière. Il y a, dans l'univers du discours de la science objective, des choses, et en premier lieu la subjectivité elle-même, qui doivent rester non dites et même impossibles à dire. C'est à une philosophie vraiment transcendentale d'entreprendre la tâche ardue de dire à sa façon ce non-dit et cet indicible. Il ne manque pas de traités sur la syntaxe logique du discours scientifique, mais quand il s'agit de dire ce qui, scientifiquement, est indicible, on se trouve dans l'embarras et dans le non-frayé. Pour dire cet indicible, c'est une nouvelle grammaire, c'est-à-dire toute une transformation du langage naturel (langage de tous les jours et langage scientifique) dont aurait besoin la philosophie. Tout progrès de la science - et c'est ici que réside le véritable rapport entre science et philosophie - finit par imposer à la philosophie du sujet, à la philosophie transcendentale, l'obligation de purifier toujours davantage son langage... et ses intentions: l'idée qu'elle se fait de ce qu'elle veut dire, c'est-à-dire qui dépasse l'ordre empirique des choses et qui - pour doter d'un nom ce qui est en fin de compte innommable - est la transcendance même. La philosophie n'est peut-être rien d'autre, toutes choses dites, que la tentative sans cesse renouvelée de dire ce qui reste toujours à dire ${ }^{61}$.

McMaster University

61. Dans cet article j'ai essayé de justifier l'entreprise de la philosophie réflexive et transcendentale et démontrer sa nécessité. Ce serait une autre question que de savoir comment le philosophe devrait parler, une fois admis qu'il doit le faire. J'ai essayé de fournir quelques éléments d'une réponse $\grave{a}$ cette autre question sur la nature d'un langage proprement philosophique, transcendental dans mon article «The Possibility and Limits of a Science of Man», à paraitre dans The Pbilosopby Fortum, no. 4, iuin, 1974. 